\title{
Electro-optic modulation in bulk silicon using surface plasmon resonance
}

Kapil Debnath ${ }^{1}$, Pedro Damas ${ }^{2}$, Liam O'Faolain*

SUPA, School of Physics \& Astronomy, University of St Andrews, North Haugh, St Andrews KY16 9SS, UK

*Corresponding author: jww1@st-andrews.ac.uk

\footnotetext{
${ }^{1}$ Nano Research Group, Faculty of Physical Sciences and Engineering, University of Southampton, Southampton, SO17 1BJ, UK, email: K.Debnath@soton.ac.uk

${ }^{2}$ Institut d'Electronique Fondamentale, Univ. Paris Sud, CNRS UMR 8622, 91405 Orsay, France, email: pedro.damas@u-psud.fr
} 


\begin{abstract}
We propose and present simulated results for a new design of an optical modulator based on Surface Plasmon Polariton (SPP) resonance. The modulator is realized on a bulk silicon substrate, thus offering an opportunity for front-end integration with electronic circuits. The device consists of a dielectric waveguide evanescently coupled to a SPP mode at the interface between bulk silicon and metal. By using SPP resonance we achieved an ultra-high spectral sensitivity ( $5000 \mathrm{~nm} /$ refractive index unit) with large modulation bandwidth $(90 \mathrm{~nm})$. For a refractive index change of 0.02 , we achieved $100 \mathrm{~nm}$ shift in resonance wavelength and a modulation depth of $\sim 10 \mathrm{~dB}$.
\end{abstract}

Keywords: Surface plasmons, Integrated optoelectronic circuits, Modulators, Silicon photonics

\title{
1. Introduction
}

As the clock speed and wiring density in microprocessors continues to rise, various problems associated with metallic interconnects are becoming increasing difficult to resolve [1-3]. The limited capacity of electrical interconnects is becoming a major problem not only for backplanes and communication between boards, but also for shorter distances, such as between chips and even onchip [4]. The physical advantages of optics over electrical signals for interconnections has heralded the emergence of the optical interconnect. Unlike an electrical wire, the limit on data transmission in an optical waveguide can be as high as 100Tbit/s and data transfer at high bit rates is much more energy efficient. At the same time, the possibility of integrating both electronics and photonics on the same platform gave rise to the field of silicon photonics $[4,5]$.

Silicon on insulator (SOI) wafers comprising of a thick buried oxide (BOx) layer have become the most popular platform for monolithic integration of silicon photonic devices with passive optical components including ultra-low loss waveguides [6] and active optical components such as high speed electro-optic modulators [7] and photodetectors [8]. However, on cost and performance grounds, bulk silicon is used in most electronic applications, such as microprocessors, high volume and high density memories etc. BOx layers are generally avoided in bulk silicon devices, as they would act as heat traps and lead to a reduction in the integration density. Therefore, in order to replace electrical interconnects with integrated photonic links for intra-chip and inter-chip communications, photonic components need to be integrated with CMOS devices on bulk silicon [9].

\section{Prior art and device design}

To achieve a single platform for photonics and electronics, different techniques have been proposed. One option is the use of multilayer structure to integrate multiple layers of photonics circuits on top of the electronic layer [10]. Another technique proposes to realize photonic components along with the electronic devices in the same plane using deep trench isolation process [11]. In both these techniques, the material platform is altered to achieve monolithic opto-electronic integration.

In this work, we propose to overcome this material incompatibility by introducing a novel modulator design based on Surface Plasmon Polariton (SPP) resonance. SPP modes are hybrid electron-photon oscillations propagating at the interface between a dielectric and a conductor. These modes are evanescently confined in the perpendicular direction, which enables the guidance of light in a twolayer structure, whereas common dielectric waveguides need at least three layers to guide light. This property of SPP waveguide opens up the opportunity to create light guiding mechanisms directly in bulk silicon without the need for any lower cladding. A 3D view of the possible implementation of 
our design is shown in Fig. 1(a). The cross sectional view is shown in Fig. 1(b). In this design the light is guided between bulk silicon and metal layer and thus obviates the need for any bottom cladding layer and achieve integration on the same plane. This implementation is remarkably simple allowing front-end integration with minimal changes to the CMOS processes currently in use. In this configuration, light propagating through the dielectric waveguide in a transverse magnetic (TM) mode evanescently couples to the SPP mode existing at the interface of the metal and bulk silicon. The coupling wavelength is decided by the intersection of the dispersion curves of the waveguide mode and the SPP mode, i.e. where both the modes are phase matched. By altering the refractive index of the silicon (e.g. by using free carrier plasma dispersion effect) we can achieve modulation at the coupling wavelength, and thus get modulation in the output intensity of the waveguide.

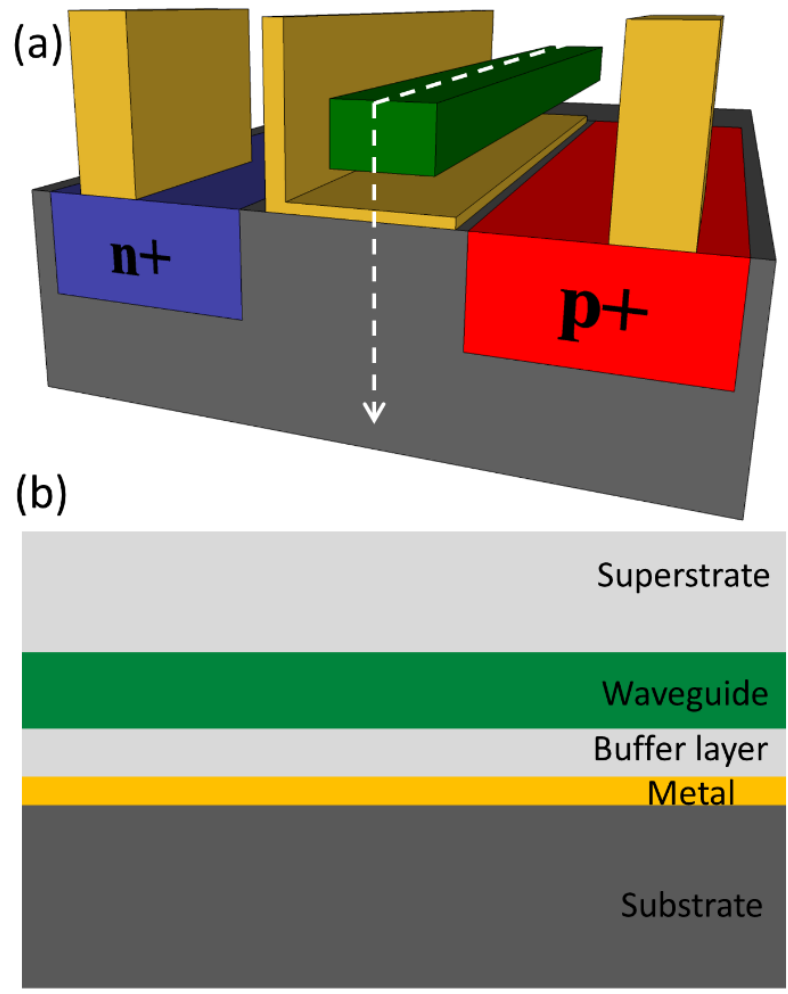

Fig. 1. (a) Schematic of the proposed SPP resonance based modulator. The structure can be embedded in a p-i-n junction as shown and the SPP resonance wavelength can be modulated using free carrier plasma dispersion effect. (b) A cross sectional view of the device. The SPP mode exists at the metal-silicon interface. The dielectric waveguide mode couples evanescently to the SP mode through the buffer layer.

\section{Simulation and results}

It is known that in refractive index sensors extraordinarily high spectral sensitivity can be achieved using SPP modes [12]. The spectral sensitivity of such sensors can be more than $10^{4} \mathrm{~nm} / \mathrm{RIU}$. When this type of device is employed as a modulator, the high spectral sensitivity means that it is capable of broadband modulation with low power consumption. In Fig. 2 we show how SPP resonances can give ultra-high spectral sensitivity. The blue curve is the dispersion relation of an SPP mode between an arbitrary semi-infinite metal and a dielectric medium. When a small positive refractive index perturbation is introduced in the dielectric region below the metal, the dispersion relation of the SPP mode shifts from the blue curve to the red curve. The black curve shows the dispersion relation of a 
dielectric waveguide. At a certain propagation constant ( $\beta$ ), the frequency (f) shift of the surface plasmon mode has a spectral sensitivity comparable with those relying on a conventional resonant cavity scheme $(\Delta \Omega)$. However, since the position of the resonance dip is determined by the intersection between the dispersion curves of the SPP mode and waveguide mode, the new resonance dip is located at a frequency associated with a different SPP mode with a different propagation constant, thus resulting in a frequency shift of $\Delta \omega>>\Delta \Omega$, This results in a large spectral sensitivity.
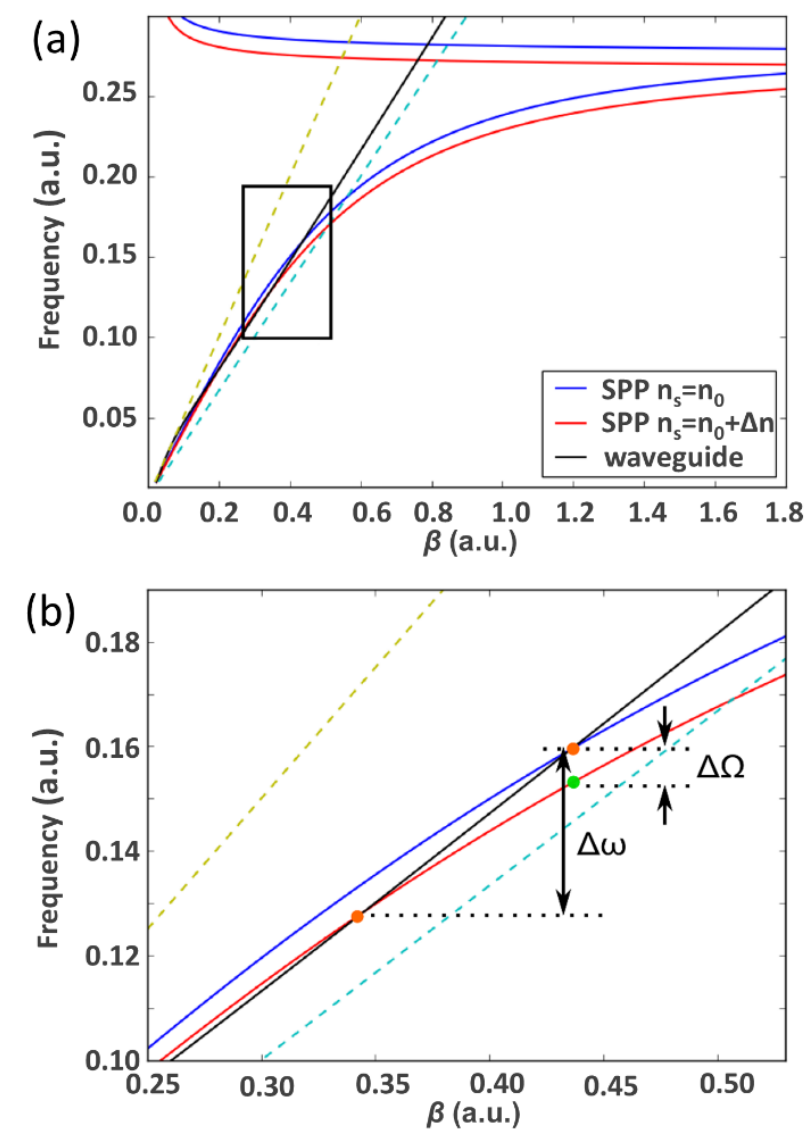

Fig. 2. (a) Dispersion relations of the waveguide mode, SPP modes with and without modified refractive index of the dielectric layer are shown in black, red and blue curves respectively. The dashed lines represent the light lines of the dielectric waveguide core and cladding. (b) Section of the dispersion relation highlighted by the black rectangle in Fig. 2(a). The orange dots show the phase matching points between two arbitrary waveguide (black) and SPP (red and blue) modes dispersion relations, whereas, the green dot shows the shift in frequency for the same propagation constant of the SPP mode. When a small change is induced in the refractive index of the dielectric

layer, a large shift in the coupling (or phase-matching) wavelength is observed, i.e. $\Delta \omega \gg>\Delta \Omega$.

Here we exploit this extraordinary sensitivity inherent to SPP in the other way round: instead of sensing a change in $\Delta n$, one can induce a change $\Delta \lambda$ in the coupling wavelength by properly changing $\Delta \mathrm{n}$ underneath the metal. The design is carried out using MEEP a freely available finite difference time domain (FDTD) simulation software [13]. We first analytically estimate the phase matching condition needed in order to couple light from the waveguide to the SPP, around the telecommunication wavelength. And then using 2D FDTD we further analyse the device properties and demonstrate the optical modulation in this structure. The structure is a 5 layers system depicted in Fig. 1(b). The substrate is bulk silicon with refractive index of 3.47 and the metal we chose is copper $(\mathrm{Cu})$. Here we avoid the use of gold or silver, the preferred metals for SPP, due to CMOS 
compatibility issues. The choice of materials for remaining layers (i.e. waveguide, buffer and superstrate) primarily depends on the effective index of the SPP mode at around 1550nm wavelength. The dispersion relation of the SPP mode at the Si-Cu interface is calculated using the Drude-Lorentz model [14] and is shown in Fig. 3.

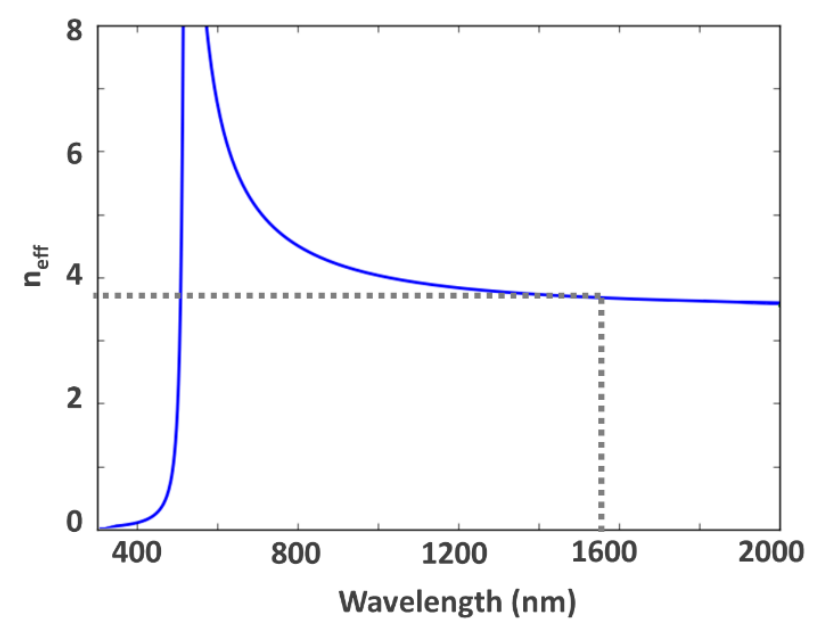

Fig. 3. Effective index of the SPP mode at the Si-Cu interface. The grey dashed line highlights the effective index of the waveguide required for coupling around the C-band of the telecomm wavelength.

The dispersion relation suggests that in order to couple light to the SPP mode around $1550 \mathrm{~nm}$ wavelength, the effective index of the dielectric waveguide mode should be close to 3.68 to ensure phase matching condition between SPP and waveguide mode. In order to achieve such high effective index we chose the waveguide refractive index to be 4.1 and for buffer and superstrate layer we chose silicon nitride (Si3N4) with refractive index of 2. Although there are not many materials available with such high refractive index in telecomm wavelength, it has been shown that with complete amorphization, amorphous-silicon can reach refractive index of 4 [15]. Here we chose the metal layer thickness of $50 \mathrm{~nm}$, the waveguide thickness of $370 \mathrm{~nm}$ and the buffer layer thickness of $150 \mathrm{~nm}$. For these dimensions the effective index of the fundamental TM mode in the waveguide has an effective index of 3.63, which corresponds to a coupling wavelength near $1600 \mathrm{~nm}$.

In order to simulate the transmission spectrum of the device, we place a pulsed (Gaussian) light source at the beginning of the waveguide and with the same cross section as the waveguide layer. The pulse is centred at $1500 \mathrm{~nm}$ with $3 \mathrm{~dB}$ bandwidth of $500 \mathrm{~nm}$. Since the metal layer is only $50 \mathrm{~nm}$ thick, we used a computational grid resolution of $10 \mathrm{~nm}$. This resolution is sufficiently high to detect plasmonic interaction in the metal. The normalized transmission spectra of the structure are shown in Fig. 4(a) for different device length and buffer layer thicknesses. For all the spectra there is a dip in transmission at around $1600 \mathrm{~nm}$. This dip is due to the coupling from the waveguide to the SPP mode. As the length of the device gets longer, the transmission dip also gets deeper suggesting more light getting coupled to the SPP mode. We also note that for the same device length as the width of the buffer region increases the dip gets shallower, while the loss also decreases, showing a lower interaction of the optical mode with the metallic layer. Figure 4(b) shows the magnetic field distribution at 1500nm (off-resonance) and $1602 \mathrm{~nm}$ (on-resonance) wavelength. On resonance, light completely couples to the SPP mode and couples back to the waveguide mode, whereas at off resonance, there is no significant coupling takes place. From the on-resonance field distribution we estimate that for complete transfer of energy the required coupling length is $130 \mu \mathrm{m}$. 


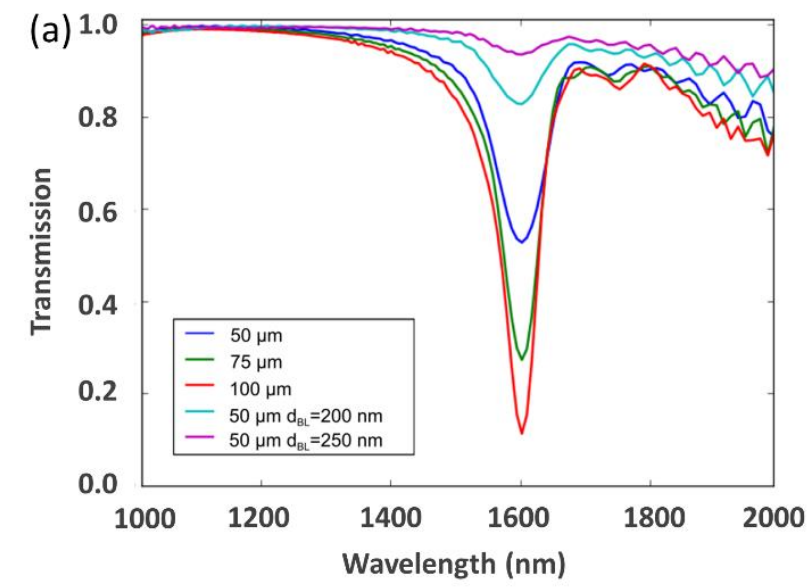

(b)

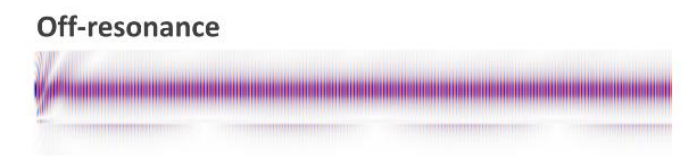

On-resonance

Fig. 4. (a) Transmission spectra of the device for different device length and buffer layer thickness. $\mathrm{dBL}$ is the buffer layer thickness. (b) Magnetic field profile at wavelengths far from the resonance $(1500 \mathrm{~nm})$ and at resonance respectively $(1602 \mathrm{~nm})$.

Finally, to demonstrate modulation we modify the refractive index of bulk silicon from 3.47 to 3.45 . Both the spectra are shown in Fig. 5. Due to the change in refractive index of the bulk silicon we can see a large shift in the resonance dip. Thus for a refractive index change of 0.02 we observe a resonance wavelength shift of $100 \mathrm{~nm}$ and a modulation depth of $\sim 10 \mathrm{~dB}$. This shift in resonance corresponds to $S \approx 5000 \mathrm{~nm} / \mathrm{RIU}$, demonstrating an ultra-high spectral sensitivity of the device. Along with ultra-high spectral sensitivity, this modulation technique offers a large modulation bandwidth, unique to this technique. For this device the full width at half maxima (FWHM) bandwidth is $90 \mathrm{~nm}$. In fact, the narrower the angle between the dispersion curves of the two modes at the phase matching point are, the greater the coupling bandwidth between the SPP and the waveguide mode (see Fig. 2). It is worth noting that for a $10 \mathrm{~dB}$ extinction ratio, the on-state loss is less than $0.5 \mathrm{~dB}$. However for on-chip communication sub $3 \mathrm{~dB}$ extinction ratios are often sufficient. In such a case, the on-state loss increases to around $3 \mathrm{~dB}$ assuming the modulator is modulated around the resonance wavelength; a value comparable to resonance based modulators [16]. 


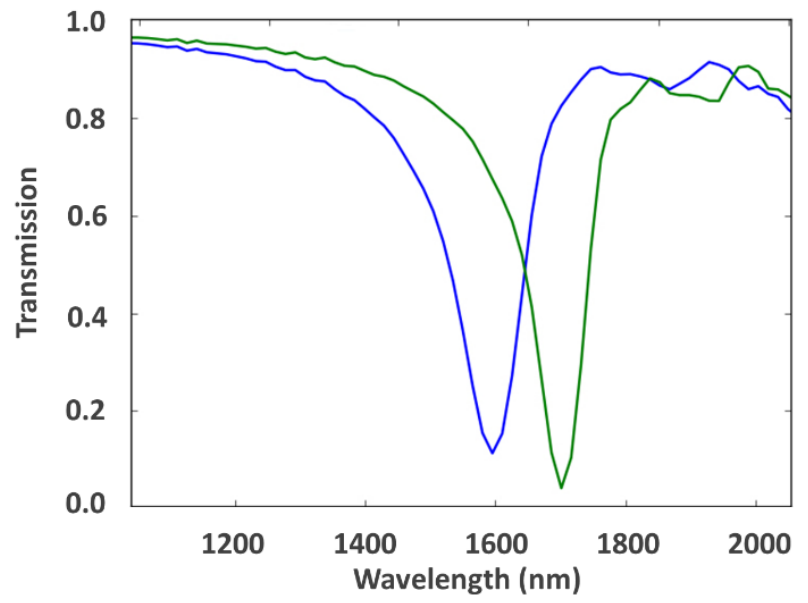

Fig. 5. Transmission spectra when the bulk silicon refractive index is 3.47 (blue) and 3.45 (green). For a refractive index change of 0.02 , the resonance wavelength shifts by $100 \mathrm{~nm}$.

\section{Conclusion}

In conclusion, we have proposed an optical modulator design based on SPP resonance due to evanescent coupling between a dielectric waveguide mode and SPP mode at the bulk silicon-metal interface. The proposed design is completely CMOS compatible and offers an easy route for frontend opto-electronic integration. Besides the obvious advantage of compatibility, the use of SPP resonance also provides ultra-high spectral sensitivity with a large modulation band-width. These characteristics are very hard to find in any optical modulator implemented so far, and gives this modulator design an enormous potential for future applications in silicon photonics and overcomes many of the limitations of modern optical modulators and communications.

\section{Acknowledgement}

The authors acknowledge funding from the EPSRC in the UK under the UK Silicon Photonics project.

\section{References}

1. R. Ho, K.W. Mai, and M.A. Horowitz, The future of wires, Proc. of the IEEE 89 (2001) 490-504.

2. D.A.B. Miller and H.M. Ozaktas, Limit to the Bit-Rate Capacity of Electrical Interconnects from the Aspect Ratio of the System Architecture, J. of Parallel and Distributed Computing 41 (1997) 42-45.

3. D.A.B. Miller, Rationale and challenges for optical interconnects to electronic chips, Proc. of the IEEE 88 (2000) 728-749.

4. D. A. B. Miller, Device Requirements for Optical Interconnects to Silicon Chips, Proc. of the IEEE 97 (2009) 1166-1185.

5. C. Batten, A. Joshi, J. Orcutt, A. Khilo, B. Moss, C. W. Holzwarth, M. A. Popović, H. Li, H. I. Smith, J. L. Hoyt, F. X. Kärtner, R. J. Ram, V. Stojanović, and K. Asanović, Building Manycore Processor-toDRAM Networks with Monolithic Silicon Photonics, IEEE symposium on High Performance Interconnects (2008) 21-30. 
6. J. Cardenas, C. B. Poitras, J. T. Robinson, K. Preston, L. Chen, and M. Lipson, Low loss etchless silicon photonic waveguides, Opt. Exp. 17 (2009) 4752-4757.

7. D. J. Thomson, F. Y. Gardes, J.-M. Fedeli, S. Zlatanovic, Y. Hu, B. P. P. Kuo, E. Myslivets, N. Alic, S. Radic, G. Z. Mashanovich, G. T. Reed, 50-Gb/s silicon optical modulator, IEEE Photon. Technol. Lett. 24 (2012) 234-236.

8. D. Marris-Morini, L. Virot, C. Baudot, J.-M. Fédéli, G. Rasigade, D. Perez-Galacho, J.-M. Hartmann, S. Olivier, P. Brindel, P. Crozat, F. Bœuf, and L. Vivien, A $40 \mathrm{Gbit} / \mathrm{s}$ optical link on a 300-mm silicon platform, Opt. Exp. 22 (2014) 6674-6679.

9. C. Sun, M. Georgas, J. Orcutt, B. Moss, Y. Chen, J. Shainline, M. Wade, K. Mehta, K. Nammari, E. Timurdogan, D. Miller, O. Tehar-Zahav, Z. Sternberg, J. Leu, J. Chong, R. Bafrali, G. Sandhu, M. Watts, R. Meade, M. Popović, R. Ram, and V. Stojanović, A monolithically-integrated chip-to-chip optical link in bulk CMOS, IEEE J. Solid state circuits 50 (2014) 828-844.

10. A. Biberman, K. Preston, G. Hendry, N. Sherwood-Droz, J. Chan, J. S. Levy, M. Lipson, and K. Bergman, Photonic network-on-chip architectures using multilayer deposited silicon materials for high-performance chip multiprocessors, ACM J. Emerg. Technol. 7 (2011) 1.

11. K. K. Mehta, J. S. Orcutt, J. M. Shainline, O. Tehar-Zahav, Z. Sternberg, R. Meade, M. A. Popović, and R. J. Ram, Polycrystalline silicon ring resonator photodiodes in a bulk complementary metaloxide-semiconductor process, Opt. let. 39 (2014) 1061-1064.

12. I. M. White and X. Fan, On the performance quantification of resonant refractive index sensors, Opt. Exp. 16 (2008) 1020-1028.

13. A. F. Oskooi, D. Roundy, M. Ibanescu, P. Bermel, J. D. Joannopoulos, and S. G. Johnson, MEEP: A flexible free-software package for electromagnetic simulations by the FDTD method, Comput. Phys. Commun. 181 (2010) 687-702.

14. A. D. Rakić, A. B. Djurišić, J. M. Elazar, and Marian L. Majewski, “Optical properties of metallic films for vertical-cavity optoelectronic devices," Appl. Opt. 37 (1998) 5271-5283.

15. R. Loiacono, G. T. Reed, G. Z. Mashanovich, R. Gwilliam, S. J. Henley, Y. Hu, R. Feldesh, and R. Jones, Laser erasable implanted gratings for integrated silicon photonics, Opt. Exp. 19 (2011) 1072810734.

16. Q. Xu, B. Schmidt, S. Pradhan, and M. Lipson, "Micrometre-scale silicon electro-optic modulator," Nature 435 (2005), 325-327. 